\title{
Reliability Allocation in Probabilistic Design Optimization of Decomposed Systems Using Analytical Target Cascading
}

\author{
Michael Kokkolaras* \\ Department of Mechanical Engineering, University of Michigan, Ann Arbor
}

\begin{abstract}
Analytical target cascading (ATC) is a methodology for translating system design targets to design specifications for the elements comprising a decomposed engineering system. In previous work we extended the ATC formulation to reliability-based design optimization (RBDO) problems, where the bounds of the probabilistic design constraints for each subproblem were assigned arbitrarily and held fixed during the ATC process. In this work, we extend the probabilistic ATC formulation to include reliability targets in the cascaded quantities. We employ a series-system formulation to solve the RBDO problem associated with each element of the ATC hierarchy and to compute its reliability. Thus, we quantify the optimality-reliability tradeoffs for each element of the decomposed system, which allows us to determine the probabilistic constraint bounds required to satisfy the overall system reliability target. In this manner we cascade the latter to element reliability specifications.
\end{abstract}

\section{Introduction}

In many industry branches including aerospace optimal system design problems appear in a decomposed form: the design optimization tasks associated with the elements that comprise the system are distributed among departments or outsourced to suppliers. In this case, smooth system integration can only be ensured when the interactions among elements are taken into account and appropriate design specifications are obtained for all subproblems.

Analytical target cascading (ATC), is a methodology for cascading system design targets to element design specifications. ${ }^{1}$ Using the multilevel hierarchy of the decomposed system, an optimization problem is formulated and solved for each element to minimize deviations of local responses from propagated targets. Solving the subproblems using appropriate coordination strategies yields overall system optimality and consistency. Several case studies have demonstrated the usefulness of ATC, e.g., in automotive engineering applications. $^{2,3}$

In recent work, we extended the deterministic ATC formulation to probabilistic design in order to account for uncertainties. ${ }^{4}$ Uncertain quantities were modeled as random variables, and we used the means of the random variables as optimization variables. The ATC optimization problems were reformulated as reliabilitybased design optimization (RBDO) problems. In RBDO, reliability is defined as the statistical estimate of satisfying probabilistically formulated design constraints. Although reliability is a system design objective of primary importance, probability of failure targets for these constraints are assigned more or less arbitrarily. For example, the vast majority of RBDO methods require the specification of reliability targets $R_{t_{p}}$ for each of the $M$ probabilistic design constraints:

$$
\begin{array}{rc}
\min _{\mu_{\mathbf{X}}} & f\left(\mu_{\mathbf{X}}\right) \\
\text { subject to } & P\left[g_{p}(\mathbf{X}) \leq 0\right]=R_{p} \geq R_{t_{p}} \quad p=1,2, \ldots, M,
\end{array}
$$

where $\mu_{\mathbf{X}}$ is the vector of the means of the random variables $\mathbf{X}, P[\cdot]$ denotes probability measure and $R$ reliability. Two recently reported RBDO methods consider an aggregated system reliability constraint that

*Associate Research Scientist, mk@umich.edu, AIAA Senior Member 
allows flexibility regarding the satisfaction of the probabilistic design constraints as long as the overall system reliability target is met. ${ }^{5,6}$ These formulations consider the probabilistic optimal system design problem as a series system since the violation of just one of these constraints would correspond to a system failure. These publications do not consider decomposition-based system design optimization, and thus do not address the reliability target allocation problem.

Reliability allocation has been studied recently for parallel-series systems in the IEEE community.7,8 A parallel-series system consists of $n$ subsystems arranged in series, each of which is comprised by $m_{i}$ parallel components, where $i=1,2, \ldots, n$. Given reliability target values $R_{t_{i}}$ for each subsystem $i$, a cost minimization problem is formulated to determine the optimal number of components $m_{i} \forall i$ and the required component reliability values $R_{t_{i j}}$, where $j=1,2, \ldots, m_{i}$ (redundancy and reliability allocation). Cost functions that are monotonic with respect to reliability were used, and it was shown that all parallel components within a subsystem must have identical reliability. These studies, however, are not concerned with design optimization. Furthermore, the system decomposition structure is limited to that of a parallelseries system. Thus, the desired system reliability can not be "translated" to required reliability targets for the elements of any arbitrary decomposition.

In this paper, we propose a multilevel optimization methodology for allocating reliability targets to the subsystems, components, etc. that comprise a system whose design is being optimized under uncertainty. Specifically, we extend the probabilistic formulation of analytical target cascading methodology to include reliability in the vector of cascaded target values. Essentially, we introduce rigorous (i.e., mathematically formal) negotiations among the elements of the multilevel hierarchy regarding their required reliability. That means that we need to compute the reliability of each element taking into account both the probabilistic design constraints associate with that element and the reliability of its "children" elements. To that end, we employ the series system approach of Ref. ${ }^{6}$ to solve the RBDO problem associated with each element of the decomposed system.

The paper is organized as follows. Section II provides a brief review of the deterministic and probabilistic analytical target cascading formulations. Section III provides a description of the series system RBDO approach that will be used to compute the reliability of each element of the decomposed system. Section IV introduces the proposed methodology for reliability target allocation. Section V presents a simple yet illustrative example used to demonstrate the proposed methodology, and Section VI provides concluding remarks.

\section{Analytical Target Cascading}

Given a hierarchical decomposition such as the one in Figure 1, analytical target cascading (ATC) is a mathematical methodology for translating ("cascading") the pre-described system design targets to design specifications for all the subsystems, components, etc. that comprise the system. The objective is to

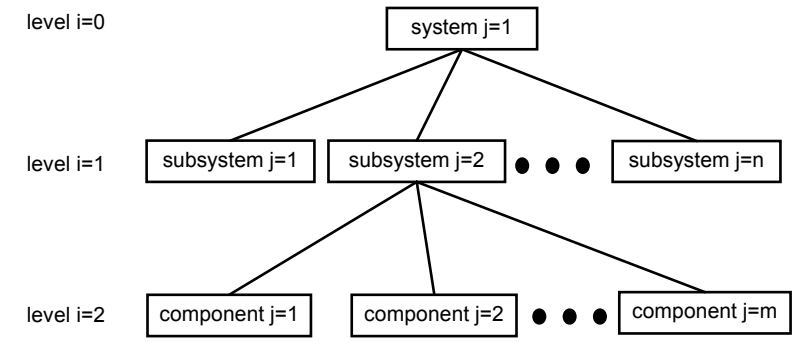

Figure 1. Three-level example of hierarchical multilevel decomposition

identify early in the design development process the relations and possible trade-offs among elements, and to determine specifications that yield consistent system design with minimized deviation from design targets. The ATC process is proven to be convergent when using a specific class of coordination strategies, ${ }^{9}$ and has been applied successfully to a variety of optimal design problems, e.g., Refs. ${ }^{10,11}$ We refer the reader to these references for a detailed description of ATC. Here, we will briefly present the concept and the general mathematical formulation. 
ATC operates by formulating and solving a minimum deviation optimization problem for each element in the hierarchy. Assuming that responses of higher level elements are functions of responses of lower-level elements, it aims at minimizing the gap between what upper-level elements "want" and what lower-level elements "can". Similarly, if design variables are shared among some elements at the same level, their consistency is coordinated by their common parent element at the level above. Assuming that element $j$ at level $i$ has $n_{i j}$ children, the aforementioned functional dependency is expressed as

$$
\mathbf{z}_{i j}=\mathbf{f}_{i j}\left(\mathbf{z}_{(i+1) 1}, \ldots, \mathbf{z}_{(i+1) n_{i j}}, \mathbf{x}_{i j}, \mathbf{y}_{i j}\right),
$$

where $\mathbf{z}_{i j}$ are element's responses, $\mathbf{z}_{(i+1) 1}, \ldots, \mathbf{z}_{(i+1) n_{i j}}$ denote children responses, $\mathbf{x}_{i j}$ represent local design variables, and $\mathbf{y}_{i j}$ denote local shared design variables (i.e., design variables that this element shares with other elements at the same level). The mathematical formulation of problem $p_{i j}$ for element $j$ at level $i$ is

$$
\begin{array}{rc}
\min & \left\|\mathbf{z}_{i j}\left(\mathbf{z}_{(i+1) 1}, \ldots, \mathbf{z}_{(i+1) n_{i j}}, \mathbf{x}_{i j}, \mathbf{y}_{i j}\right)-\mathbf{z}_{i j}^{u}\right\|_{2}^{2}+\left\|\mathbf{y}_{i j}-\mathbf{y}_{i j}^{u}\right\|_{2}^{2}+ \\
& \sum_{k=1}^{n_{i j}}\left\|\mathbf{z}_{(i+1) k}-\mathbf{z}_{(i+1) k}^{l}\right\|_{2}^{2}+\sum_{k=1}^{n_{i j}}\left\|\mathbf{y}_{(i+1) k}-\mathbf{y}_{(i+1) k}^{l}\right\|_{2}^{2} \\
\text { with respect to } & \mathbf{z}_{(i+1) 1}, \ldots, \mathbf{z}_{(i+1) n_{i j}}, \mathbf{x}_{i j}, \mathbf{y}_{i j}, \mathbf{y}_{(i+1) 1}, \ldots, \mathbf{y}_{(i+1) n_{i j}} \\
\text { subject to } & \mathbf{g}_{i j}\left(\mathbf{z}_{i j}, \mathbf{x}_{i j}, \mathbf{y}_{i j}\right) \leq \mathbf{0},
\end{array}
$$

where coordinating variables for the shared design variables of the children are denoted by $\mathbf{y}_{(i+1) 1}, \ldots, \mathbf{y}_{(i+1) n_{i j}}$, and local design constraint functions are represented by $\mathbf{g}_{i j}$. Superscripts $u(l)$ are used to denote response and shared variable values that have been obtained at the parent (children) problem(s), and have been cascaded down (passed up) as design targets (consistency parameters), $c f$. Figure 2. Note that although com-

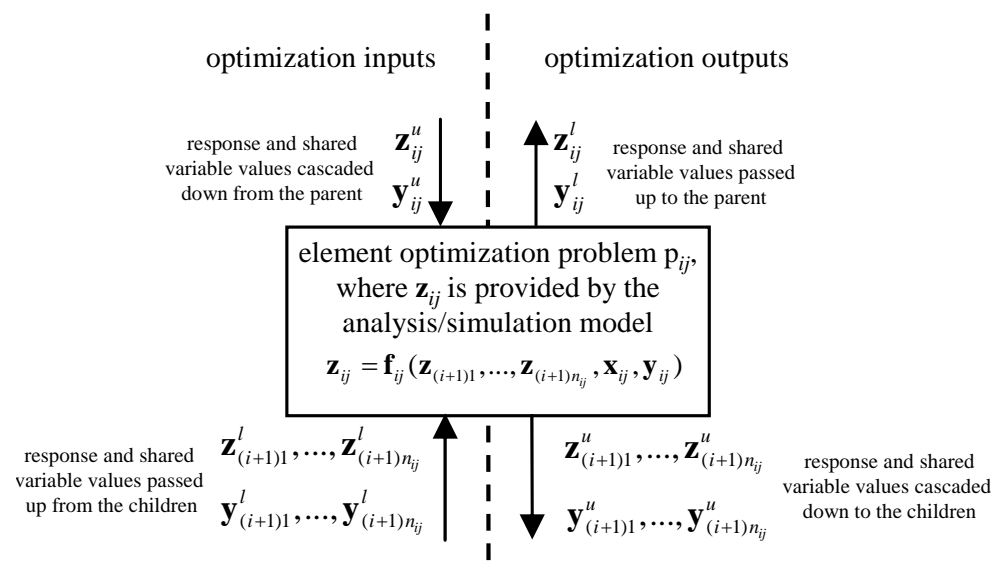

Figure 2. ATC information flow at element $j$ of level $i$

munication among levels, i.e., updating parameter values associated with the ATC process, is bi-directional, functional dependency is strictly hierarchical. Assuming that all the element's problem parameters have been updated using the solutions obtained at the parent- and children-problems, Problem (3) is solved to update the parameters of the parent- and children-problems. All the subproblems are solved iteratively according to an appropriate coordination strategy until optimization variables converge (i.e., don't change anymore).

\section{A. Probabilistic ATC Formulation}

The deterministic ATC formulation assumes that complete information of the system design problem is available, and that design decisions can be implemented precisely. These assumptions imply that optimization results are as good (and therefore useful) as the design and simulation/analysis models used to obtain them, and that they are meaningful only if they can be realized exactly. In reality, these assumptions do not hold. We are rarely in a position to represent a physical system without using approximations, have complete knowledge on all of its parameters, or control the design variables with high accuracy. It is therefore imperative to represent uncertainties and take them into account during the early design assessment process.

The ATC formulation has been extended to account for uncertain quantities by modeling them as random variables (denoted by upper case Latin symbols). ${ }^{4}$ The means of the random variables are treated as 
optimization variables. Functions of random variables are approximated by their expected value, and design constraints are formulated probabilistically, as in Problem (1). The probabilistic ATC formulation is

$$
\begin{array}{rc}
\min & \left\|\mu_{\mathbf{Z}_{i j}}\left(\mu_{\mathbf{Z}_{(i+1) 1}}, \ldots, \mu_{\mathbf{Z}_{(i+1) n_{i j}}}, \mu_{\mathbf{X}_{i j}}, \mu_{\mathbf{Y}_{i j}}\right)-\mu_{\mathbf{Z}_{i j}}^{u}\right\|_{2}^{2}+\left\|\mu_{\mathbf{Y}_{i j}}-\mu_{\mathbf{Y}_{i j}}^{u}\right\|_{2}^{2}+ \\
& \sum_{k=1}^{n_{i j}}\left\|\mu_{\mathbf{Z}_{(i+1) k}}-\mu_{\mathbf{Z}_{(i+1) k}}^{l}\right\|_{2}^{2}+\sum_{k=1}^{n_{i j}}\left\|\mu_{\mathbf{Y}_{(i+1) k}}-\mu_{\mathbf{Y}_{(i+1) k}}^{l}\right\|_{2}^{2} \\
\text { with respect to } & \mu_{\mathbf{Z}_{(i+1) 1}}, \ldots, \mu_{\mathbf{Z}_{(i+1) n_{i j}}}, \mu_{\mathbf{X}_{i j}}, \mu_{\mathbf{Y}_{i j}}, \mu_{\mathbf{Y}_{(i+1) 1}}, \ldots, \mu_{\mathbf{Y}_{(i+1) n_{i j}}} \\
\text { subject to } & P\left[g_{i j, p}\left(\mathbf{Z}_{i j}, \mathbf{X}_{i j}, \mathbf{Y}_{i j}\right) \leq 0\right]=R_{i j, p} \geq R_{t_{i j, p}}, \quad p=1,2, \ldots, M_{i j}
\end{array}
$$

where $M_{i j}$ is the number of probabilistic design constraints and $R_{t_{i j, p}}$ is the desired reliability for each constraint.

\section{B. Uncertainty Propagation}

In a multilevel hierarchy, responses of lower-level elements are inputs to higher-level elements. This is an issue of utmost importance in design optimization of hierarchically decomposed systems under uncertainty, since the solution of probabilistic optimization problems requires variance information of the random optimization variables. Consider element $j$ at level $i$. By solving Problem (4), we obtain optimal values $\mu_{\mathbf{Z}_{(i+1) 1}^{*}}^{*}, \ldots, \mu_{\mathbf{Z}_{(i+1) n_{i j}}^{*}}^{*}, \mu_{\mathbf{X}_{i j}}^{*}$, and $\mu_{\mathbf{Y}_{i j}}^{*}$. Using the functional dependency relation $\mathbf{Z}_{i j}=$ $\mathbf{f}_{i j}\left(\mathbf{Z}_{(i+1) 1}, \ldots, \mathbf{Z}_{(i+1) n_{i j}}, \mathbf{X}_{i j}, \mathbf{Y}_{i j}\right)$, we must estimate the standard deviations of the responses $\mathbf{Z}_{i j}$ since they constitute parameters of the parent probabilistic optimal design problem (estimation of means is not critical because they constitute optimization variables of the parent probabilistic optimal design problem; accurate estimates provide merely good initial guesses for the optimization algorithm). This needs to be done for all problems at all levels of the hierarchy. Depending on the computational cost of the required evaluations, one can use either the Monte Carlo method, or efficient approximation methods such as those presented in. ${ }^{4,12}$

Assuming that initial uncertainty information is available at the bottom level of the hierarchy, its propagation through the hierarchy during the ATC process can be summarized as follows.

1. Starting at the bottom level of the hierarchy, where the probability density functions of the random variables are assumed to be known, solve the RBDO problems. Since parameter values from the top level are not available at this first iteration, one can use reasonable or "stretched" target values based on knowledge of the problem.

2. Obtain the equivalent means and standard deviations of the response variables that are inputs to the parent problems, and solve the latter.

3. Once the top level has been reached and the system RBDO has been solved, cascade updated targets down the hierarchy using previous solutions to update parameters.

4. Keep iterating until all optimization variables in all problems have converged (i.e., are not changing significantly anymore).

\section{Component Reliability Estimation Using Series System RBDO}

Since a component design has to satisfy all the constraints (there is no redundancy, i.e., violation of one constraint leads to failure), it can be viewed as a series system. Therefore, to solve the RBDO problem (1) for each element of the hierarchy, and estimate its reliability, we adopt the series-system RBDO method of. ${ }^{6}$ In this method, the optimizer determines not only the optimal values of the original optimization variables $\mu_{\mathbf{X}}$, but also the optimal reliability value for each probabilistic design constraint. Given a component reliability target, the optimizer allocates reliability target values among the failure failure modes (a failure mode being the violation of a probabilistic design constraint). Reliability allocation and optimal design are conducted simultaneously. The reliability values $R_{j}$ of the probabilistic design constraints are included in the set of optimization variables. We must ensure that the "constraint" reliability $R_{C}$, a function of the reliability of all constraints, exceeds the desired component reliability $R_{t}$. According to Ref. ${ }^{6}$ this constraint reliability is approximated by

$$
R_{C}=1-\left(\sum_{p=1}^{M} P_{f_{p}}-\sum_{p, q=1}^{M} \max _{q<p} P_{f_{p q}}\right)
$$


where $P_{f_{p}}$ and $P_{f_{p q}}$ represent probability of failure of constraint $p$ and joint probability of failure of constraints $p$ and $q$, respectively (where $p, q=1,2, \ldots, M$ ). Recall that reliability and probability of failure are linked through the relation $R=1-P_{f}$.

The series-system RBDO problem formulation then becomes

$$
\begin{gathered}
\min _{\mu_{\mathbf{X}}, R_{1}, R_{2}, \ldots, R_{M}} \\
\text { subject to } \\
\\
\\
R_{t}-1+\left(\sum_{p=1}^{M} P_{f_{p}}-\sum_{p, q=1}^{M} \max _{q<p} P_{f_{p q}}\right)=R_{t}-R_{C}\left(R_{1}, R_{2}, \ldots, R_{M}\right) \leq 0 .
\end{gathered}
$$

\section{Target Cascading for Reliability Allocation}

In a decomposed system, the reliability of each element (subsystem, component, etc.) is determined not only by its ability to satisfy the probabilistic design constraints (estimated using the approach presented in Section III), but also depends on the reliability of its "children" elements. Specifically, the reliability $R_{E}$ of an element with $M$ probabilistic design constraints and $N$ children is

$$
R_{E}=R_{C} \prod_{k=1}^{N} R_{E_{k}}=\left(1-\left(\sum_{p=1}^{M} P_{f_{p}}-\sum_{p, q=1}^{M} \max _{q<p} P_{f_{p q}}\right)\right) \prod_{k=1}^{N} R_{E_{k}} .
$$

Note that if an element has no children, then the product $\prod_{k=1}^{N} R_{E_{k}}$ does not show up in Eq. (7), i.e., for a "childless" element we have $R_{E}=R_{C}$.

Let us consider the generic element $j$ at level $i$ of the hierarchy ( $c f$. Figure 3). The reliability of the

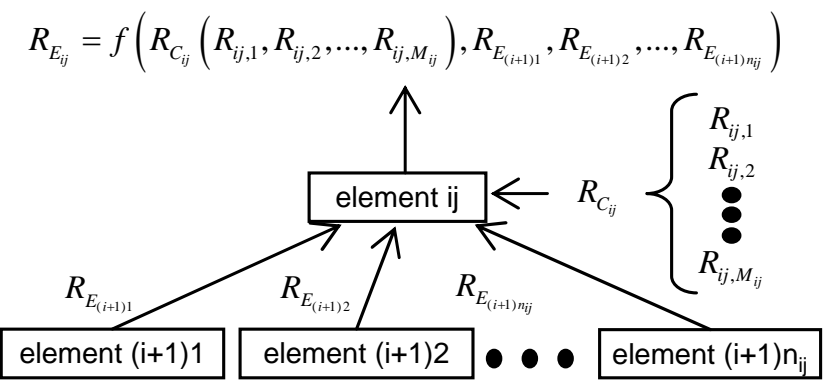

Figure 3. Reliability estimation for element $j$ at level $i$ of the hierarchy

element is a function of its "design constraint" reliability $R_{C_{i j}}$ and the children reliability values $R_{E(i+1) k}$, $k=1,2, \ldots, n_{i j}$, where $n_{i j}$ is the number of children elements. In our proposed methodology for reliability allocation, we exploit this functional dependency, and include reliability in the formulation of the ATC subproblems as follows.

$$
\begin{gathered}
\min \left\|\mu_{\mathbf{Z}_{i j}}\left(\mu_{\mathbf{Z}_{(i+1) 1}}, \ldots, \mu_{\mathbf{Z}_{(i+1) n_{i j}}}, \mu_{\mathbf{X}_{i j}}, \mu_{\mathbf{Y}_{i j}}\right)-\mu_{\mathbf{Z}_{i j}}^{u}\right\|_{2}^{2}+\sum_{k=1}^{n_{i j}}\left\|\mu_{\mathbf{Z}_{(i+1) k}}-\mu_{\mathbf{Z}_{(i+1) k}}^{l}\right\|_{2}^{2}+ \\
\left(R_{E i j}\left(R_{C_{i j}}\left(R_{i j, 1}, \ldots, R_{i j, M_{i j}}\right), R_{E_{(i+1) 1}}, \ldots, R_{E_{(i+1) n_{i j}}}\right)-R_{E_{i j}}^{u}\right)^{2}+\sum_{k=1}^{n_{i j}}\left(R_{E_{(i+1) k}}-R_{E_{(i+1) k}}^{l}\right)^{2}+ \\
\left\|\mu_{\mathbf{Y}_{i j}}-\mu_{\mathbf{Y}_{i j}}^{u}\right\|_{2}^{2}+\sum_{k=1}^{n_{i j}}\left\|\mu_{\mathbf{Y}_{(i+1) k}}-\mu_{\mathbf{Y}_{(i+1) k}}^{l}\right\|_{2}^{2} \\
\text { w.r.t. } \quad \mu_{\mathbf{Z}_{(i+1) 1}}, \ldots, \mu_{\mathbf{Z}_{(i+1) n_{i j}}}, \mu_{\mathbf{X}_{i j}}, \mu_{\mathbf{Y}_{i j}}, \mu_{\mathbf{Y}_{(i+1) 1}}, \ldots, \mu_{\mathbf{Y}_{(i+1) n_{i j}}}, R_{i j, 1}, \ldots, R_{i j, M_{i j}}, R_{(i+1) 1}, \ldots, R_{(i+1) n_{i j}} \\
\text { subject to } P\left[g_{i j_{p}}\left(\mathbf{Z}_{i j}, \mathbf{X}_{i j}, \mathbf{Y}_{i j}\right) \leq 0\right] \geq R_{i j, p}, \quad p=1,2, \ldots, M_{i j} \\
\left(1-\left(\sum_{p=1}^{M_{i j}} P_{f_{p}}-\sum_{p, q=1}^{M_{i j}} \max _{q<p} P_{f_{p q}}\right)\right) \prod_{k=1}^{n_{i j}} R_{E_{(i+1) k}} \geq R_{E_{i j}}^{u}
\end{gathered}
$$

The ATC process for reliability allocation is initiated at the bottom level, and follows the description of Section II B. Bottom-level elements do not have any children: their reliability is equal to their "design constraint" reliability, computed using the technique of Section III. These reliability values are then passed 
up to the next level, where they are used, together with local reliability values, to estimate the reliability of the elements at that level, and so on. When the top level is reached, the system ATC problem examines whether system reliability meets the desired reliability target. If necessary, the ATC problems are solved again, this time going down the hierarchy, and the whole process is repeated until convergence. Note that it is possible that the ATC process will converge without meeting top-level reliability (or other performance) targets. The ATC process does not guarantee that top-level targets will be met; it guarantees that design specifications and reliability targets will be allocated to all elements of the decomposed system so that the design of the latter is consistent while trying to meet the pre-specified top-level system design and reliability targets as close as possible.

\section{Example}

In this section, we demonstrate the methodology for reliability allocation using a two-level example, which is a slight modification of the problem presented in Ref. ${ }^{1}$ The deterministic formulation of the three subproblems of the two-level decomposed optimization problem are presented in Figure 4.

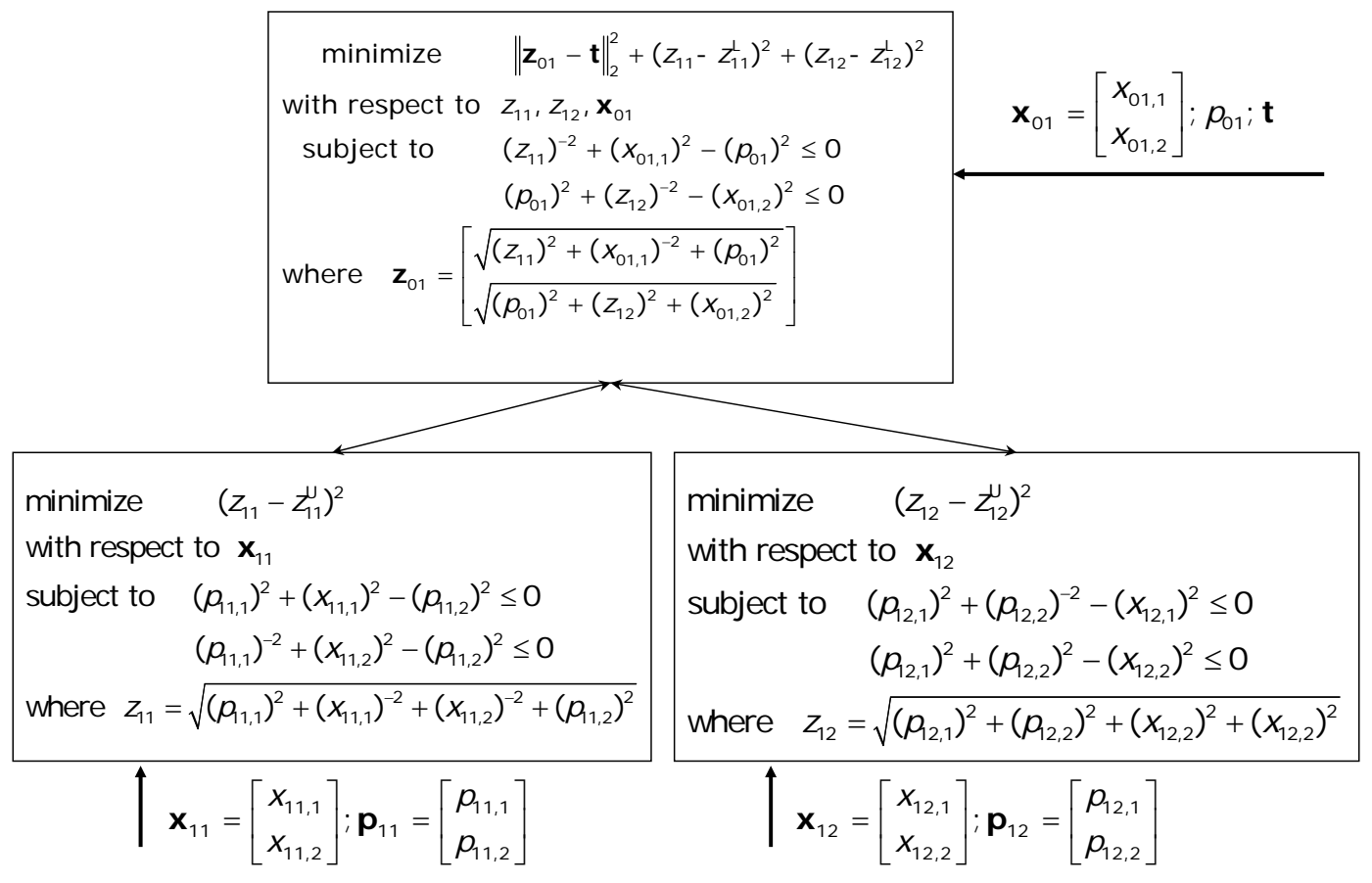

Figure 4. Deterministic formulation of the two-level optimization problem

Let us now consider the case where there is uncertainty in the parameters $p_{01}, p_{11,1}$ and $p_{12,2}$ (modeled as normally distributed random variables $P_{01}, P_{11,1}$ and $\left.P_{12,2}\right)$, which propagates through the hierarchy since all responses and design constraints in bottom-level optimization problems depend on these parameters, and because all responses and design constraints in the top-level optimization problem depend on bottom-level responses. The RBDO formulation of the two-level problem with arbitrarily assigned reliability target values for the probabilistic constraints is presented in Figure 5.

Introducing the reliability target variables into the ATC process using the series-system approach, as described in Section IV, yields the two-level reliability allocation problem shown in Figure 6.

We obtained results for the following parameter values of the deterministic optimization problem

$$
\mathbf{t}=\left[\begin{array}{ll}
0 & 0
\end{array}\right]^{\mathrm{T}} ; \quad p_{01}=0.87 \quad ; \quad \mathbf{p}_{11}=\left[\begin{array}{ll}
0.97 & 1.3
\end{array}\right]^{\mathrm{T}} \quad ; \quad \mathbf{p}_{\mathbf{1 2}}=\left[\begin{array}{ll}
1.3 & 0.84
\end{array}\right]^{\mathrm{T}}
$$

while in the probabilistic optimization problem we considered a $5 \%$ coefficient of variation for each uncertain parameter and required a top-level system reliability $R_{\mathrm{T}}$ of 0.9 (or $90 \%$ percent). Table 1 summarizes the obtained results. We used the Monte Carlo method to solve the reliability analysis problem for each 


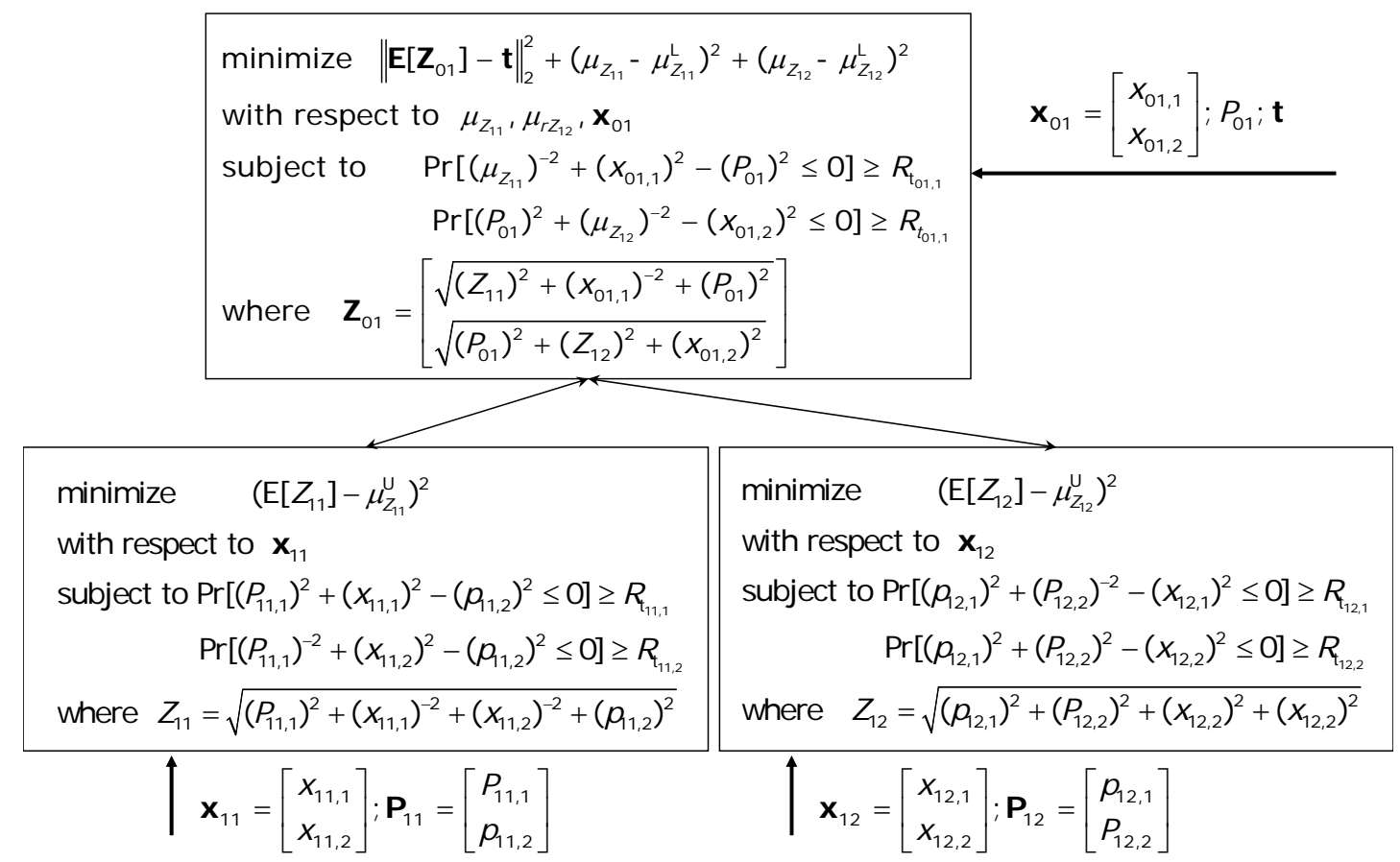

Figure 5. RBDO formulation of the two-level optimization problem with arbitrarily assigned reliability targets for the probabilistic constraints

probabilistic constraint in the optimization subproblems. We can see how uncertainty affects the solution of the problem and increase the objective function value of the problem, since it is expected that, to accommodate uncertainty, the optimal solution changes to the detriment of optimality. The significant part of the results is that we have obtained the required reliability targets for the probabilistic design constraints of the bottom-level problems, and thus the required element reliability targets for these two subsystems, as well as the required reliability target values for the probabilistic design constraints of the top-level problem so that the system reliability target is met. These results are summarized in Table 2, which includes the verifying calculations. Note that the system reliability $R_{E_{01}}$ meets the assigned target $R_{\mathrm{T}}$ exactly.

\section{Concluding Remarks}

We have presented an extension of the probabilistic formulation of Analytical Target Cascading (ATC) to conduct reliability target allocation in decomposition-based system optimal design under uncertainty. We have assumed that each element of the multilevel hierarchy that represents the decomposed system is a series system, whose reliability depends on satisfying all probabilistic design constraints as well as on the reliability of all its "children" elements. We have demonstrated the applicability of the proposed methodology using a simple analytical example. Future work will investigate issues such as approximations made during element reliability analysis and uniqueness of solutions.

\section{Acknowledgments}

The author is grateful to Dr. Zissimos Mourelatos of Oakland University for many helpful and insightful discussions and suggestions. This work was partially supported by the Automotive Research Center, a US Army Center of Excellence at the University of Michigan. Any opinions expressed in this publication are only those of the author. 


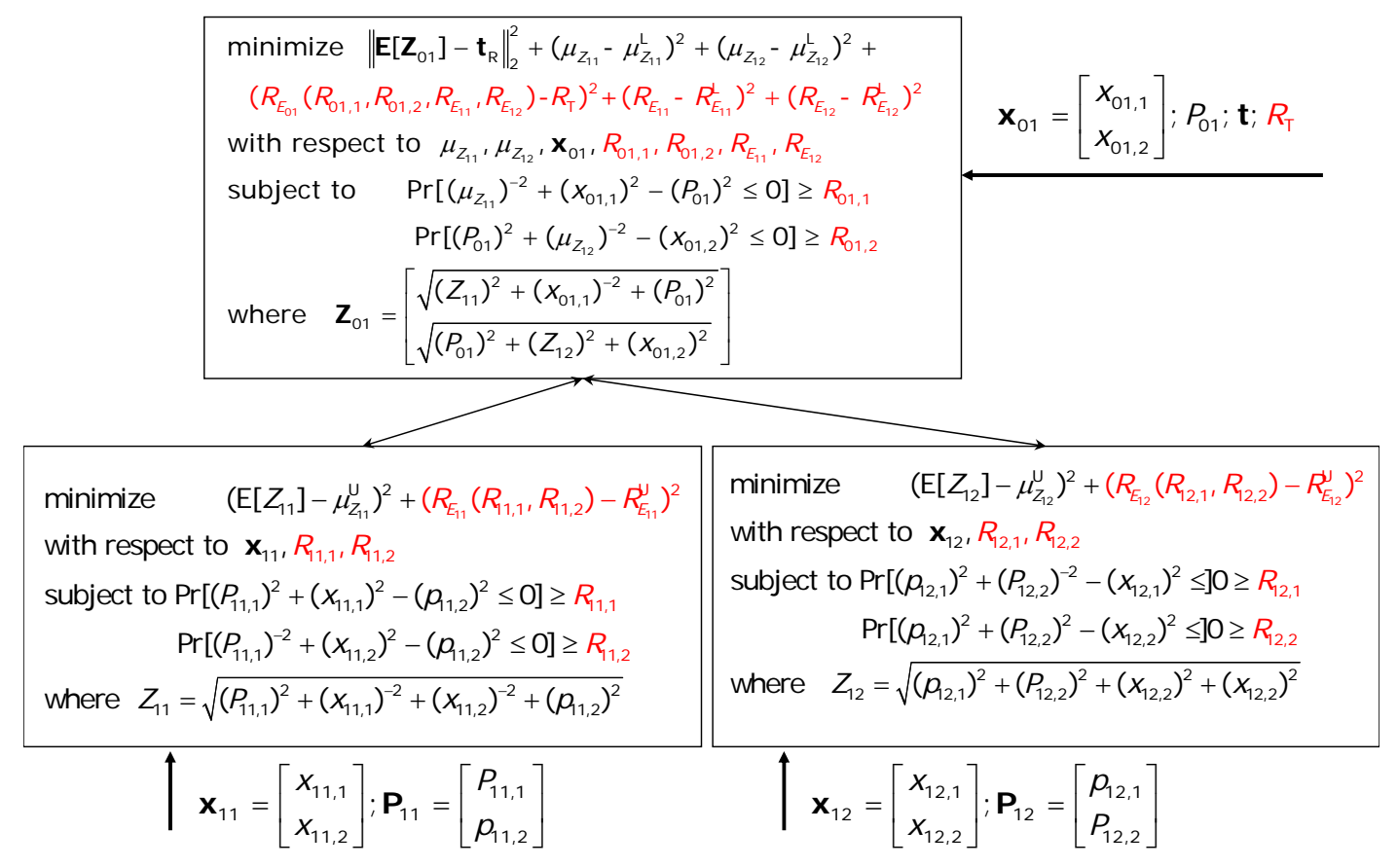

Figure 6. Reliability allocation formulation of the two-level optimization problem using ATC

\section{References}

${ }^{1}$ Kim, H., Michelena, N., Papalambros, P., and Jiang, T., "Target Cascading in Optimal System Design," ASME Journal of Mechanical Design, Vol. 125, No. 3, 2003, pp. 474-480.

${ }^{2}$ Kim, H., Kokkolaras, M., Louca, L., Delagrammatikas, G., Michelena, N., Filipi, Z., Papalambros, P., Stein, J., and Assanis, D., "Target Cascading in Vehicle Redesign: A Class VI Truck Study," International Journal of Vehicle Design, Vol. 29, No. 3, 2002, pp. 1-27.

${ }^{3}$ Kokkolaras, M., Louca, L., Delagrammatikas, G., Michelena, N., Filipi, Z., Papalambros, P., Stein, J., and Assanis, D., "Simulation-Based Optimal Design of Heavy Trucks by Model-Based Decomposition: An Extensive Analytical Target Cascading Case Study," International Journal of Heavy Vehicle Systems, Vol. 11, No. 3-4, 2004, pp. 402-432.

${ }^{4}$ Kokkolaras, M., Mourelatos, Z., and Papalambros, P., "Design Optimization of Hierarchically Decomposed Multilevel Systems under Uncertainty," Journal of Mechanical Design, Vol. 12, No. 2, 2006, pp. 502-508.

${ }^{5}$ Chan, K.-Y., Skerlos, S., and Papalambros, P., "A Single-Loop Approach for System Reliability-Based Design Optimization," Proceedings of the 32nd ASME Design Automation Conference, Philadelphia, Pennsylvania, September 10-13, 2006, paper no. DETC2006-DAC99417.

${ }^{6}$ Liang, J., Mourelatos, Z., and Nikolaidis, E., "A Single-Loop Approach for System Reliability-Based Design Optimization," Proceedings of the 32nd ASME Design Automation Conference, Philadelphia, Pennsylvania, September 10-13, 2006, paper no. DETC2006-DAC99240.

${ }^{7}$ Elegbede, A., Chu, C., Adjallah, K., and Yalaoui, F., "Reliability Allocation Through Cost Minimization," IEEE Transactions on Reliability, Vol. 52, No. 1, 2003, pp. 106-111.

${ }^{8}$ Yalaoui, A., Chatelet, E., and Chu, C., "'A New Dynamic Programming Method for Reliability \& Redundancy Allocation in a Parallel-Series System," IEEE Transactions on Reliability, Vol. 54, No. 2, 2005, pp. 254-261.

${ }^{9}$ Michelena, N., Park, H., and Papalambros, P., "Convergence Properties of Analytical Target Cascading," AIAA Journal, Vol. 41, No. 5, 2003, pp. 897-905.

${ }^{10}$ Kokkolaras, M., Fellini, R., Kim, H., Michelena, N., and Papalambros, P., "Extension of the Target Cascading Formulation to the Design of Product Families," Structural and Multidisciplinary Optimization, Vol. 24, No. 4, 2002, pp. $293-301$.

${ }^{11}$ Kim, H., Rideout, D., Papalambros, P., and Stein, J., "Analytical Target Cascading in Automotive Vehicle Design," ASME Journal of Mechanical Design, Vol. 125, No. 3, 2003, pp. 481-489.

${ }^{12}$ Youn, B., Kokkolaras, M., Mourelatos, Z., Papalambros, P., Choi, K., and Gorsich, D., "Uncertainty Propagation Techniques for Probabilistic Design of Multilevel Systems," Proceedings of the 10th AIAA/ISSMO Sumposium on Multidisciplinary Analysis and Optimization, Albany, New York, August 30 - September 1, 2004, paper no. AIAA-2004-4470. 
Table 1. Results for the deterministic and probabilistic formulations of the two-level optimization problem

\begin{tabular}{|c|c|c|}
\hline Response or design variable & Deterministic solution & Probabilistic solution \\
\hline \hline$z_{01,1}\left(E\left[Z_{01,1}\right]\right.$ in probabilistic case $)$ & 2.80 & 3.29 \\
\hline$z_{01,2}\left(E\left[Z_{01,2}\right]\right.$ in probabilistic case $)$ & 3.03 & 3.21 \\
\hline$x_{01,1}$ & 0.76 & 0.71 \\
\hline$x_{01,2}$ & 0.95 & 1.02 \\
\hline$z_{11}\left(E\left[Z_{11}\right]\right.$ in probabilistic case $)$ & 2.35 & 2.85 \\
\hline$x_{11,1}$ & 0.87 & 0.69 \\
\hline$x_{11,2}$ & 0.80 & 0.55 \\
\hline$z_{12}\left(E\left[Z_{12}\right]\right.$ in probabilistic case $)$ & 2.79 & 2.91 \\
\hline$x_{12,1}$ & 1.75 & 1.87 \\
\hline$x_{12,2}$ & 1.54 & 1.61 \\
\hline
\end{tabular}

Table 2. Reliability allocation results and verifying calculations

\begin{tabular}{|c|c|c|}
\hline Reliability & Value & Verifying calculation (when applicable) \\
\hline \hline$R_{01,1}$ & 0.940 & \\
$R_{01,2}$ & 0.980 & \\
\hline$R_{11,1}$ & 0.991 & \\
$R_{11,2}$ & 0.999 & \\
\hline$R_{12,1}$ & 0.984 & \\
$R_{12,2}$ & 0.998 & $=1-(0.009+0.001-0)$ \\
\hline$R_{E_{11}}=R_{C_{11}}$ & 0.990 & $=1-\left(\operatorname{Pr}\left[g_{11,1}>0\right]+\operatorname{Pr}\left[g_{11,2}>0\right]-\operatorname{Pr}\left[g_{11,1}>0\right.\right.$ AND $\left.\left.g_{11,2}>0\right]\right)$ \\
& & $=1-\left(\operatorname{Pr}\left[g_{12,1}>0\right]+\operatorname{Pr}\left[g_{12,2}>0\right]-\operatorname{Pr}\left[g_{12,1}>0\right.\right.$ AND $\left.\left.g_{12,2}>0\right]\right)$ \\
$R_{E_{12}}=R_{C_{12}}$ & 0.982 & $=1-(0.016+0.002-0)$ \\
& & $=1-\left(\operatorname{Pr}\left[g_{0,1}>0\right]+\operatorname{Pr}\left[g_{0,2}>0\right]-\operatorname{Pr}\left[g_{0,1}>0\right.\right.$ AND $\left.\left.g_{0,2}>0\right]\right)$ \\
$R_{C_{01}}$ & 0.920 & $=1-(0.06+0.02-0)$ \\
\hline$R_{E_{01}}$ & 0.900 & $=R_{C_{01}} R_{E_{11}} R_{E_{12}}=0.920 \cdot 0.990 \cdot 0.982$ \\
\hline
\end{tabular}

